\title{
The Quantum Characters of Two-level-atom to the Cavity Optomechanical system
}

\author{
Kun Yang ${ }^{1, a}$, Dingyun $\mathrm{Jin}^{2, \mathrm{~b}}$ and Xin Sui ${ }^{3, \mathrm{C}}$ \\ ${ }^{1,2,3}$ changchun engineering technology college, Changchun, 130117, China \\ a,b,c email: 294244450@qq.com
}

Keywords: The cavity optomechanical system; two-level-atom; quantum characters

\begin{abstract}
With the widely application and development of the mechanical system and optical microcavity, the strong coupling between mirror and cavityfield in the system of cavity optomechanics, that make the optomechanical system become an important platform which is used to inspect macroscopic quantum phenomena and text quantum theory, so that become a new research hot spot. At the same time, for cavity opto-mechanics, there are important application prospect in gravitational waves detection, infinitesimal is placement and the precision measurement of the quality and so on, therefore the study of cavity opto-mechanics has the profound significance. This article first introduced the optical mechanical cavity, and then examines the atomic population is poor. We found that the difference of atomic population with the time evolution law of motion state of the mirror is very sensitive, if the initial state of the mirror as the coherent state, the atomic population difference will collapse and reply phenomenon, collapse time and cycle depends on the cavity photon number and average number of vibrator. Finally studied the optical mechanical two-level atomic emission spectrum characteristics of cavity.
\end{abstract}

\section{Introduction}

In recent years, with the continuous development of optical microcavity and electromechanical systems, optical radiation field in the cavity and the coupling between the mechanical system has attracted widespread attention in the field of quantum engineering, optical mechanical system is similar to the existence of the electromagnetic induction phenomenon of transparent, with pressure cavity to improve radiation cooling, the micro mechanical oscillator is also using reaction to the micro mechanical oscillator cooling to the ground state, which laid the foundation for the development of quantum technology.

Jaynes - Cummings (jaynes-cummings model is the study of a single atom and single mode the ideal model of quantum field interaction, it is one of the models can be calculated precisely in quantum optics, it can be used to explain many phenomena in quantum optics, people conducted in-depth research on it and the corresponding promotion, such as three level atom with a single-mode light field, the interaction between the system of atoms interacting with multi-mode light field, multiple atoms and the interaction of light field system, multiphoton jaynes-cummings model, nonlinear jaynes-cummings model and so on, in this paper, the research of light machinery of the chamber system under certain conditions can be simplified as generalized jaynes-cummings model.

\section{The cavity optomechanical system}

the light coupling with mechanical vibrator can change the frequency of the cavity field, will also affect the dynamic behavior of vibrator in the cavity optomechanical System. The cavity optomechanical is a kind of the light field coupling of the optical cavity, it is composed of A fixed mirror A and A connected with spring can do tiny vibration of the mirror side mirror (activities) B. A two-level atom in the cavity optomechanical as shown in figure 1. When A beam of laser driver by A mirror when incident light mechanical cavity, the cavity on the activities side mirror B radiation pressure, B in the small mechanical vibration of the equilibrium position of the change the effective length of cavity, make the space of the cavity field intensity distribution and light cavity frequency modulation, and the change of the cavity field is bound to affect the interaction of atoms. On the other 
hand, the optical cavity atomic radiation will affect the distribution of activities will also change the cavity end mirror vibration.

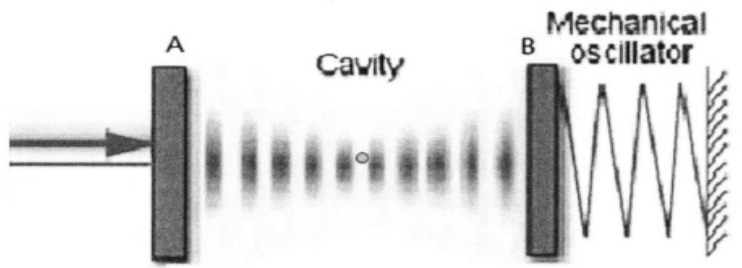

Figure1. A and B constitute A mirror light mechanical cavity, B is connected to A spring, lumen built-in two level atom

\section{The Two-level Atomic Occupancy}

Interact with light field of atoms will reflect the quantum properties of light field. Among them, collapse and reply phenomenon of the two-level atomic occupancy is a typical example. J-C model can describe the ideal light field in the interaction of two-level atoms and the cavity system in the interaction Hamiltonian is in matte painting $\mathrm{HJC}=\mathrm{HJC} 0+\mathrm{VJC}$.

If the light field in the photon number state $|n\rangle, F_{n^{\prime}}=\delta_{n, n}$. When in a coherent state light field $<\mathrm{Sz}$ (t) $>$ first presents the collapse phenomenon, after a long time period of stability is 0 , then began to reply. After doing the periodic collapse and reply, reply cycle associated with the $<\mathrm{n}>$ of light field.

The reply period is $2 \pi \sqrt{\langle n\rangle} / g$, but the collapse of the time is not sensitive to the average photon number $<\mathrm{n}>$, about $(2 \mathrm{~g})-1$. As shown in figure 2 .
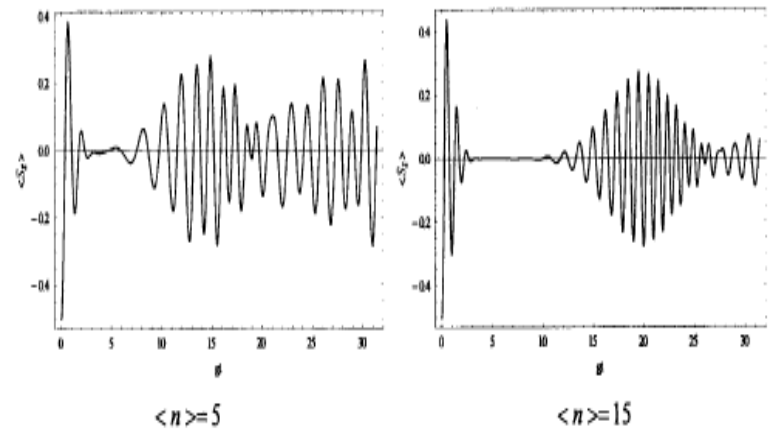

Figure 2. $<\mathrm{Sz}(\mathrm{t})>$ evolution with time, the light field in a coherent state

\section{The Characteristics of the Two-level Atomic Emission Spectrum}

Assuming that light field and oscillator are photon coherent state in the cavity optomechanical system. Average photon number of the cavity is $<\mathrm{n}>$, the average number of vibrator is $<\mathrm{m}>$ and $\eta=1, \tau=0.09 \eta, T=30 / \eta$. In the cavity optomechanical system, When photon coherent state cavity field in the add, oscillator in possession when the number of coherent states, emission spectrum of a two-level atom is shown in figure 3.

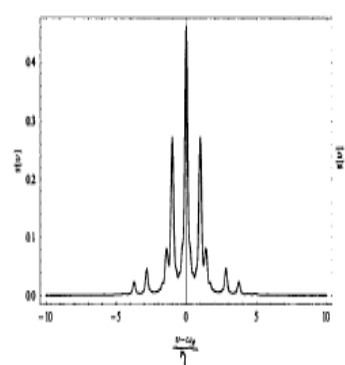

$i=0, j=0,|\alpha|^{2}=1,|\beta|^{2}=1$

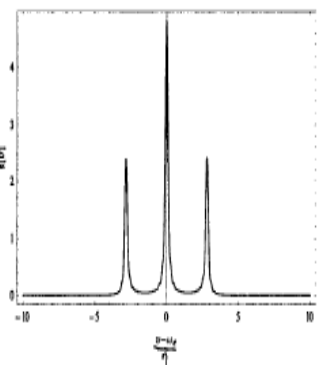

$i=1, j=1, \alpha=0, \beta=0$

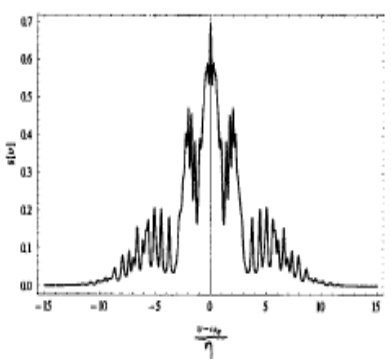

$i=0, j=0,|\alpha|^{2}=1,|\beta|^{2}=4$

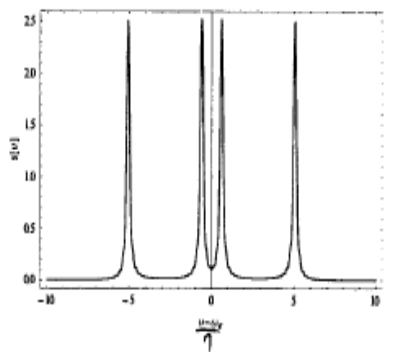

$i=1, j=4, \alpha=0, \beta=0$

Fig.3 Left:oscillator is in a coherent state cavity field of atomic emission spectrum. Right: the cavity in the photon number state, the oscillator in possession of several states of atomic emission spectrum. 
As shown in figure 3 on the right side, if the cavity in the photon number state, if the oscillator in possession of several states at the same time, the emission spectrum of a two-level atom is simple three or four peak peak shape. And about the atomic transition frequency $(\mathrm{v}-\mathrm{wc})$ is symmetrical,

spectrum peak position is in

$$
\begin{aligned}
& \omega_{e} \pm \eta(\sqrt{(1+<m>)<n>}+\sqrt{\langle m\rangle(1+\langle n\rangle)} \\
& \omega_{e} \pm \eta(\sqrt{(1+<m>)<n>}-\sqrt{\langle m\rangle(1+<n>)}
\end{aligned}
$$

. And outside the side peaks decreases with the distance between the photon number and the increment of the vibrator possess several wide.

As shown in figure 3 on the left side, if in a coherent state cavity field, if the vibrator is in a coherent state, the two-level atomic emission spectrum at the transition frequency presents unimodal or bimodal symmetry. Than, equal to zero when the atomic emission spectrum, frequency of transition spectral line at this time in a pair of symmetrical edge on either side of the unimodal or bimodal peak into a symmetrical peaks concentrated area, and the peak concentration area with the increase of the average photon number or average number and move to each side and wider range of peak height decreases.

If the optical field of photon number unchanged, oscillator and particle number is the same, and the average photon number is equal to the average number, atomic emission spectrum at the transition frequency in May, with the average photon number or the increase of the average number of vibrator was chopped ChengShuangFeng single-peak line, and the peak height decreases: both sides of the emission spectrum presents two symmetrical wave agglomeration area, with the increase of the average photon number two peaks concentrated area to the lateral movement, and wider area range, peak height decreases. Coherent state cavity field average photon number change when atomic emission spectrum as shown in figure 4.

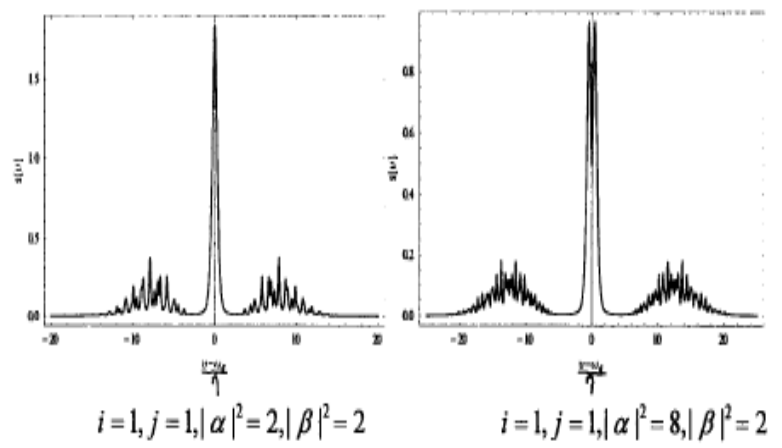

Figure 4. coherent state cavity field average photon number change when atomic emission spectrum If light field with the increase of number of photons, optical field average photon number increased as well. The average photon number is equal to the average number, were no longer unimodal gradually evolved into symmetric doublet. Transition frequency peaks on both sides of the gathered group also with light field or oscillator is becoming more and more irregular with the increase of number of photons. For symmetry evolve unimodal and range is more and more wide. Plus photon number, the greater the whole curve is the closer for particle number state light field. Atomic emission spectrum of cavity field and photon number change is shown in figure 5 .

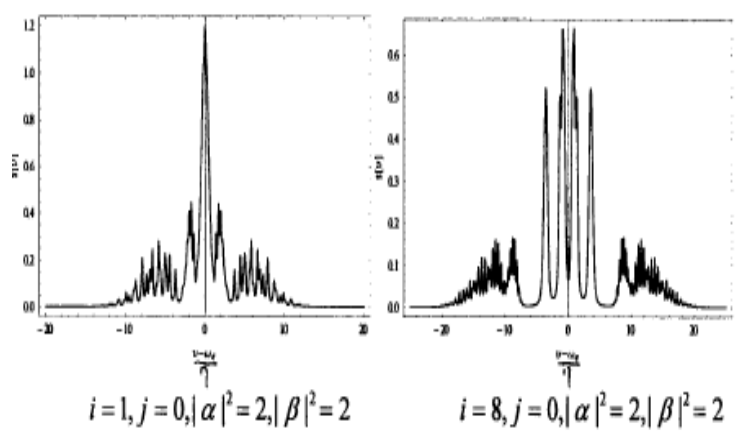

Figure 5. atomic emission spectrum of cavity field and photon number change 
Two-level atomic emission spectrum at the transition frequency $(\mathrm{v}-\mathrm{wc})$ appeared unimodal reason is when the average photon number of the light field is equal to the average number of vibrator, oscillator and light field took the chance to state of maximal amplitude of particle number $\mathrm{m}$ is equal to the photon number $\mathrm{n}$, they overlap a unimodal - wc (v), and when they are not equal to will be split into a bimodal symmetrical about (v - wc). For the coherent state light field or oscillator, the state is by several superpositions, around the average number of particles. By the different light field or oscillator state in probability of different wave function, so it is a series of spectral peak peak different wave superposition. Hence spectral lines will appear in the symmetrical about (v - wc) wave agglomeration area. Atomic emission spectrum of oscillator coherent states the average number of changes is shown in figure 6 .
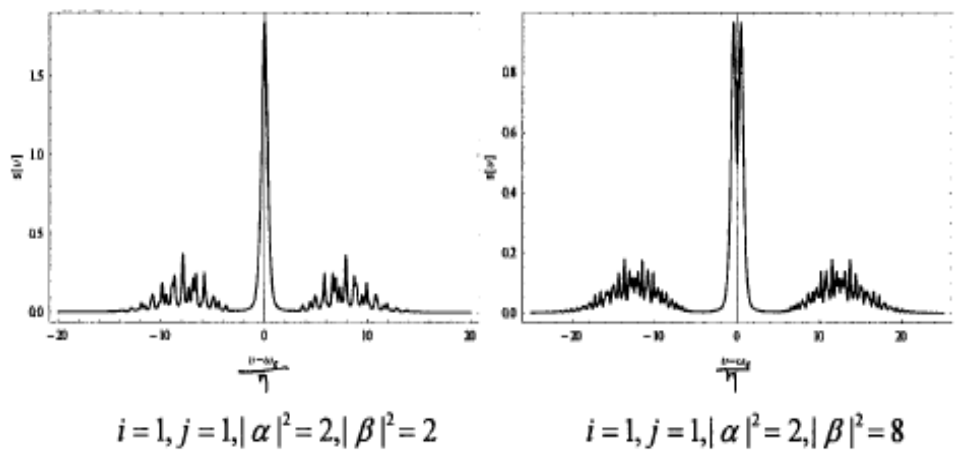

Figure 6. atomic emission spectrum of oscillator coherent states the average number of changes

When the light field or oscillator coherent state coefficient $\alpha, \beta$ increases, will make its coherence enhancement, atomic emission spectrum will increasingly tend to be coherent state of emission spectrum.And when the light field and the photon number or oscillator of particle number increases, will make its particles, atomic emission spectrum is more and more tend to the emission spectrum of particle number state. Atomic emission spectrum of oscillator add number changes is shown in figure 7.
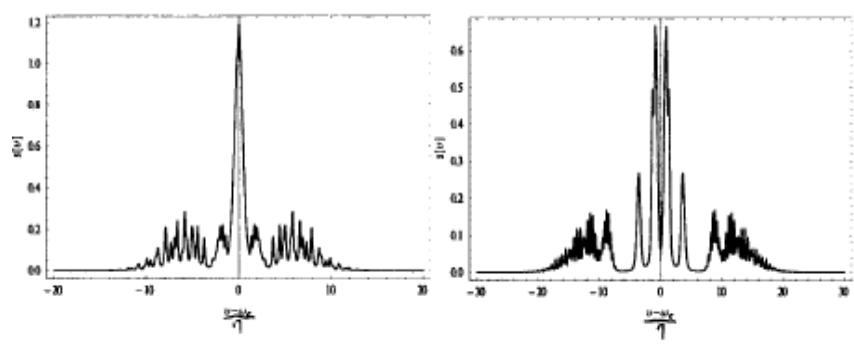

$$
i=0, j=1,|\alpha|^{2}=2,|\beta|^{2}=2
$$$$
i=0, j=8,|\alpha|^{2}=2,|\beta|^{2}=2
$$

Figure7. Atomic emission spectrum of oscillator add number changes

\section{Conclusion}

The interaction process of atom and light field in the cavity optomechanical system is still hot spot for researching. This article studies still need to further deepen. This paper deal with the problem when assuming vibrator do not associated with light field, ignored in the center of mass movement of the atom, it doesn't take into account loss of system, therefore, we can further study oscillator and the connection degree of light field, atom loss of center of mass sports, the system of atom and light field in optical mechanical cavity interaction process.

\section{References}

[1] Xue. xi YiflournalofMoeden Optics,43: 4, 675(2009).

[2] Jing H, Li Y and Zhan MS, Chin.Phys.16 1883(2007).

[3] Tang P and Guo H, Chinese Physics B18(08)(2009). 